\title{
Critical Points of the Ginzburg-Landau Functional on Multiply-Connected Domains
}

\author{
J. W. Neuberger and R. J. Renka
}

\section{CONTENTS}

1. Introduction

2. Discretization and Sobolev Gradient

3. Numerical Methods

4. Results

Acknowledgements

References

Keywords: Ginzburg-Landau functional, superconductivity, Sobolev gradient

AMS Subject Classification: 35J50, 65K10,81V99
We give a numerical method for approximating critical points of the Ginzburg-Landau functional, and present test results in the form of plots of the corresponding electron densities, magnetic fields, and currents. Our domains include a rectangle, a rectangle with a rectangular hole in the center, and a rectangle with two rectangular holes. In each case, we found several critical points. The plots reveal interesting patterns, including the existence of counter-currents (adjacent currents in opposite directions).

\section{INTRODUCTION}

There is considerable current interest, both mathematical and physical, in Ginzburg-Landau energy functionals. The critical points of these functionals represent distributions of currents and magnetic fields in superconducting materials. Superconductors have a number of important industrial applications, including high-speed semiconductor devices with low power consumption. Numerical computation of critical points is essential for two purposes: to provide simulations for use by designers of superconducting devices, and to provide guidance to mathematicians seeking to characterize the critical points. It is the latter purpose we emphasize here, with particular attention to finding multiple critical points of a given functional, and to the behavior of currents and magnetic fields in domains with holes.

Let $\Omega$ be a bounded, simply connected region in $\mathbb{R}^{2}$, inside which we consider zero or more holes, whose union we denote by $\Omega_{0}$. The region $\Omega \backslash \Omega_{0}$ represents a cross section of a superconductor whose energy is given by the (nondimensionalized) GinzburgLandau functional, which is defined on

$$
u \in H^{1,2}(\Omega, \mathbb{C}) \quad \text { and } \quad A \in H^{1,2}\left(\Omega, \mathbb{R}^{2}\right)
$$


by the expression

$$
\begin{aligned}
& E(u, A)= \\
& \frac{1}{2} \int_{\Omega \backslash \Omega_{0}}\left(\|\nabla u-i u A\|^{2}+\left(\nabla \times A-H_{0}\right)^{2}+\frac{\kappa^{2}}{2}\left(|u|^{2}-1\right)^{2}\right) \\
& +\frac{1}{2} \int_{\Omega_{0}}\left(\nabla \times A-H_{0}\right)^{2}, \quad(1-1)
\end{aligned}
$$

We interpret $u$ as a complex wave function, referred to as the order parameter, with $|u|^{2}$ representing the (probability) density of superconducting electrons (Cooper pairs), and $A$ as the magnetic field vector potential so that $\nabla \times A$ (third component) is the induced magnetic field. The applied magnetic field $H_{0}$ is the magnetic field that would be present in the absence of the superconductor, and a local minimum of $E$ is obtained with $u=0$ and $\nabla \times A=H_{0}$ everywhere in $\Omega$. The Ginzburg-Landau parameter $\kappa$ is a temperature-independent nondimensional material number [Rubinstein 1998].

A simplified version of $(1-1)$ is treated in [Bethuel et al. 1994]:

$$
E(u)=\frac{1}{2} \int_{\Omega}\left(\|\nabla u\|^{2}+\frac{\kappa^{2}}{2}\left(|u|^{2}-1\right)^{2}\right) .
$$

A mathematical study of minimizers of this functional has been carried out in [Bethuel et al. 1994; Shafrir 1995; Comte and Mironescu 1995] as well as in papers to which these works refer. Much of that work seems to be directed toward developing intuition which might carry over to (1-1). In [Neuberger and Renka 1997] we describe numerical experiments in which we located two families of critical points for 1-2 under boundary conditions of degree $d$, for $d=2, \ldots, 10$. These were found in response to a question raised in [Bethuel et al. 1994, problem 12, p. 139].

The critical points of $E$ are solutions to the stationary Ginzburg-Landau equations - that is, the Euler equations associated with (1-1) - with the corresponding natural boundary conditions - a pair of coupled nonlinear PDE's with nonlinear boundary conditions [Du et al. 1992; Neuberger and Renka 1999]. The equations can be uncoupled and the boundary conditions can be linearized, but the cost of the computational procedure is quite high $[\mathrm{Mu}$ and Huang 1998]. A second approach to computing the critical points is to evolve the time-dependent Ginzburg-Landau equations until a steady state is reached [Du et al. 1992; 1995; Fleckinger-Pellé et al. 1998; Shafrir 1995]. This too is very costly.

We have developed a very efficient method that avoids the problem of dealing with the nonlinear boundary conditions by treating the least squares minimization problem directly. We employ a steepest descent method using a discretized Sobolev gradient in place of the standard gradient [Neuberger 1997; Neuberger and Renka 1999]. At each descent step a symmetric positive definite linear system is solved (by a multigrid method) to obtain the Sobolev gradient from the standard gradient. This descent method is effectively a damped Newton iteration with a positive definite approximation to the Hessian. The method is easily extended to three space dimensions, and it appears to be applicable to a wider class of energy functionals such as YangMills-Higgs functionals [Jaffe and Taubes 1980]. For the two-dimensional domains treated in this paper, each test run required only a few minutes on a 550 $\mathrm{MHz}$ Pentium III.

The efficiency of our method and the fact that it does not require a unique solution enabled us to compute several critical points for each of several configurations (choices of the geometry $\Omega_{0}$ and parameter values $\left.H_{0}, \kappa\right)$. We obtain different critical points simply by varying the initial estimate used by the descent method. While the existence of multiple critical points is known ([Serfaty 1999], for example), we are not aware of any other numerical studies in which multiple critical points are computed (or holes are included in the domain). The primary purpose of this paper is to provide a first step toward a complete classification of the critical points of $E$.

Since we have no way of knowing if we have computed all the critical points for any given configuration, the number of critical points remains an open question. A possible approach to the problem of classifying critical points of a functional is discussed in [Neuberger 1997, Chapter 14]. The present work, in a sense, forms a basis for a numerical implementation of the idea described in that chapter for the case of Ginzburg-Landau functionals. Call two members of $H^{1,2}(\Omega, \mathbb{C}) \times H^{1,2}\left(\Omega, \mathbb{R}^{2}\right)$ equivalent if they lead (via steepest descent with Sobolev gradients) to the same critical point of $E$. Using this definition of equivalence, one obtains a foliation, each 
leaf of which contains exactly one critical point. A topological-algebraic characterization of these leaves might lead to a solution of the problem of determining all critical points of $E$.

Although nonlinear, the Ginzburg-Landau model was derived from quantum mechanical considerations, and the plots of electron densities and magnetic fields in Figures 1-4 are similar in appearance to eigenfunctions of a Schrödinger operator associated with a sequence of energy values. A full classification of the critical points could lead to a substantial chapter in the very important but still ill-defined area of nonlinear spectral theory.

The method is described in Sections 2 and 3, and our test results are presented in Section 4.

\section{DISCRETIZATION AND SOBOLEV GRADIENT}

We begin by recasting (1-1) in terms of real-valued functions. Let $u=r+i s$ and $A=\left(\begin{array}{l}p \\ q\end{array}\right)$. Then

$$
\begin{aligned}
& E(p, q, r, s)= \\
& \frac{1}{2} \int_{\Omega \backslash \Omega_{0}}\left(\left(r_{1}+s p\right)^{2}+\left(s_{1}-r p\right)^{2}+\left(r_{2}+s q\right)^{2}\right. \\
&+\left(s_{2}-r q\right)^{2}+\left(q_{1}-p_{2}-H_{0}\right)^{2} \\
&\left.+\left((\kappa / \sqrt{2})\left(r^{2}+s^{2}-1\right)\right)^{2}\right) \\
& \quad+\frac{1}{2} \int_{\Omega_{0}}\left(q_{1}-p_{2}-H_{0}\right)^{2},
\end{aligned}
$$

where the subscripts 1 and 2 on $p, q, r$, and $s$ denote first partial derivatives with respect to the first and second arguments, respectively. Once a critical point of $(2-1)$ is found, the corresponding current is

$$
J(p, q, r, s)=\left(r^{2}+s^{2}\right)\left(\begin{array}{c}
p \\
q
\end{array}\right)-r \nabla s+s \nabla r .
$$

Let $w=\left(\begin{array}{c}r \\ s \\ p \\ q\end{array}\right)$, and define $D: H^{1,2}(\Omega)^{4} \rightarrow L_{2}(\Omega)^{12}$
\[ D w=\left(\begin{array}{c}w \\ w_{1} \\ w_{2}\end{array}\right) . \]

Then $D w(x) \in \mathbb{R}^{12}$ for $x \in \Omega$, and from $(2-1)$ there exist $F: \mathbb{R}^{12} \rightarrow \mathbb{R}^{6}$ and $F_{0}: \mathbb{R}^{12} \rightarrow \mathbb{R}$ such that

$$
\begin{aligned}
\varphi(w) \equiv E(p, q, r, s)=\frac{1}{2} \| F(D w) & \|_{L_{2}\left(\Omega \backslash \Omega_{0}\right)^{6}}^{2} \\
& +\frac{1}{2}\left\|F_{0}(D w)\right\|_{L_{2}\left(\Omega_{0}\right)}^{2} .
\end{aligned}
$$

By the Riesz Representation Theorem, the linear functional $\varphi^{\prime}(w)$ is uniquely represented by an element of $\left(H^{1,2}(\Omega)\right)^{4}$ - the Sobolev gradient $\nabla_{S} \varphi(w)$ :

$$
\varphi^{\prime}(w) h=\left\langle h, \nabla_{S} \varphi(w)\right\rangle_{\left(H^{1,2}(\Omega)\right)^{4}} \quad \text { for } h \in\left(H^{1,2}(\Omega)\right)^{4} .
$$

We now describe a discretization and construct the analogous Sobolev gradient for the corresponding finite dimensional setting. We take $\Omega$ to be the rectangle $[0, \Delta x] \times[0, \Delta y]$ and partition it into a $k_{x}$-cell by $k_{y}$-cell rectangular grid with horizontal mesh width $h_{x}=\Delta x / k_{x}$ and vertical mesh width $h_{y}=\Delta y / k_{y}$. We take $\Omega_{0}$ to be a subset of the interior grid cells. Denote the set of $\left(k_{x}+1\right)\left(k_{y}+1\right)$ grid points by $G$, and denote the set of cell centers by $G^{\prime}$. Let $H$ be the vector space of all $\mathbb{R}^{4}$-valued functions on $G$, and let $H^{\prime}$ be the set of functions from $G^{\prime}$ to $\mathbb{R}^{12}$, so that $H$ and $H^{\prime}$ are analogous to $\left(H^{1,2}(\Omega)\right)^{4}$ and $\left(L_{2}(\Omega)\right)^{12}$, respectively. We then define $D_{G}: H \rightarrow H^{\prime}$ by

$$
D_{G} w=\left(\begin{array}{c}
\widehat{I} w \\
D_{1} w \\
D_{2} w
\end{array}\right)
$$

where $\widehat{I}, D_{1}$, and $D_{2}$ map grid-point functions to cell-center functions as follows: if the cell with center $e$ has corners $a, b, c, d$ at the lower left, lower right, upper left, and upper right, respectively, then

$$
\begin{aligned}
\widehat{I} w(e) & =(w(a)+w(b)+w(c)+w(d)) / 4, \\
D_{1} w(e) & =(w(b)-w(a)+w(d)-w(c)) /\left(2 h_{x}\right), \\
D_{2} w(e) & =(w(c)-w(a)+w(d)-w(b)) /\left(2 h_{y}\right) .
\end{aligned}
$$

With this notation our discretized functional is

$$
\begin{aligned}
\varphi_{G}(w)=\frac{h_{x} h_{y}}{2}\left(\sum_{e \in G^{\prime} \cap \Omega \backslash \Omega_{0}}\left\|F\left(D_{G} w\right)(e)\right\|_{\mathbb{R}^{6}}^{2}\right. \\
\left.+\sum_{e \in G^{\prime} \cap \Omega_{0}}\left\|F_{0}\left(D_{G} w\right)(e)\right\|_{\mathbb{R}}^{2}\right),
\end{aligned}
$$

where the subscripts denote Euclidean norms on the designated spaces. To obtain a Sobolev gradient for $\varphi_{G}$, we must define a corresponding inner product for $H$. The obvious choice would be, for $v, w \in H$, $\langle v, w\rangle_{S}=\left\langle D_{G} v, D_{G} w\right\rangle_{H^{\prime}}$, where the subscript $H^{\prime}$ denotes Euclidean inner product on $H^{\prime}$ (or on $\mathbb{R}^{N}$ for $N=12 k_{x} k_{y}$ ). However, there exists $w \neq 0$ such that $D_{G} w=0$. We therefore define

$$
\langle v, w\rangle_{S}=\langle\bar{D} v, \bar{D} w\rangle_{H^{\prime \prime}} \quad \text { for } v, w \in H,
$$


where

$$
\bar{D} w=\left(\begin{array}{c}
w \\
D_{1} w \\
D_{2} w
\end{array}\right),
$$

and the $H^{\prime \prime}$ inner product is defined appropriately.

The linear functional $\varphi_{G}^{\prime}(w)$ has two representations by means of gradients. The value of the conventional gradient at $w$, denoted by $\nabla \varphi_{G}(w)$, is obtained by evaluating partial derivatives of $\varphi_{G}$ at $w$. With this gradient, the representation is

$$
\varphi_{G}^{\prime}(w) h=\left\langle h, \nabla \varphi_{G}(w)\right\rangle_{H} \quad \text { for } h \in H,
$$

where the subscript $H$ denotes the standard $\mathrm{Eu}$ clidean inner product on $H$ (or on $\mathbb{R}^{n}$ for $n=4 \times$ $\left.\left(k_{x}+1\right)\left(k_{y}+1\right)\right)$. In terms of the Sobolev gradient or $S$-gradient $\nabla_{S} \varphi_{G}$ of $\varphi_{G}$, the representation is

$$
\varphi_{G}^{\prime}(w) h=\left\langle h, \nabla_{S} \varphi_{G}(w)\right\rangle_{S} \quad \text { for } h \in H .
$$

The relationship between the two gradients is given by equations $(2-2),(2-3)$, and $(2-4)$ :

$$
\begin{array}{rlr}
\varphi_{G}^{\prime}(w) h & =\left\langle h, \nabla_{S} \varphi_{G}(w)\right\rangle_{S} & \\
& =\left\langle\bar{D} h, \bar{D} \nabla_{S} \varphi_{G}(w)\right\rangle_{H^{\prime \prime}} & \\
& =\left\langle h, \bar{D}^{t} \bar{D} \nabla_{S} \varphi_{G}(w)\right\rangle_{H} & \\
& =\left\langle h, \nabla \varphi_{G}(w)\right\rangle_{H} \quad \text { for } h \in H .
\end{array}
$$

It follows that

$$
\nabla_{S} \varphi_{G}(w)=\left(\bar{D}^{t} \bar{D}\right)^{-1} \nabla \varphi_{G}(w) \quad \text { for } w \in H .
$$

See [Neuberger 1997; Neuberger and Renka 1999] for a more extensive discussion of the properties of Sobolev gradients.

\section{NUMERICAL METHODS}

The steepest descent iteration step is

$$
w_{k+1}=w_{k}-\alpha_{k}\left(\bar{D}^{t} \bar{D}\right)^{-1} \nabla \varphi_{G}\left(w_{k}\right),
$$

where $w_{0}$ is an initial solution estimate and $\alpha_{k}$ is computed by a line search minimization of $\psi\left(\alpha_{k}\right)=$ $\varphi\left(w_{k}-\alpha_{k} \nabla_{S} \varphi_{G}\left(w_{k}\right)\right)$. We use Brent's univariate optimization algorithm [1973], which combines golden section search with parabolic interpolation.

Note that, with the Hessian of $\varphi_{G}$ at $w_{k}$ in place of $\bar{D}^{t} \bar{D},(3-1)$ becomes the Newton iteration for computing a zero of $\nabla \varphi_{G}$. The advantage of approximating the Hessian values by the symmetric positive definite operator $\bar{D}^{t} \bar{D}$ is that the matrix need not be stored, and the linear systems can be solved by a multigrid method with both storage and time complexity linear in the number of grid points. To this end we define a sequence of grids $G_{0}, G_{1}, \ldots, G_{M}$, where $G_{0}$ is as coarse as possible given the constraints on the geometry (the hole locations), and $G_{i}$ is a refinement of $G_{i-1}$ obtained by bisecting each cell both horizontally and vertically.

Write the linear system as $A u=b$, where

$$
\begin{aligned}
A & =A_{M}=\bar{D}^{t} \bar{D}, \\
u & =u_{M}=\nabla_{S} \varphi_{G_{M}}\left(w_{k}\right), . \\
b & =b_{M}=\nabla \varphi_{G_{M}}\left(w_{k}\right)
\end{aligned}
$$

The first step of the multigrid scheme is to apply one to three steps of an iterative method chosen to effectively damp out the high frequency components of the error. This step is referred to as pre-smoothing. Let $\bar{u}$ denote the approximate solution. Then the error $e=u-\bar{u}$ is the solution to $A e=r$ for residual $r=b-A \bar{u}$. The correction $e$ is approximated by restricting $r$ and $A$ to a coarser grid, solving for $e$ using 0 as the initial estimate, interpolating the solution back to the fine grid, and correcting the fine-grid solution $\bar{u}$. This coarse-grid solution step efficiently damps out the low-frequency error components. The final step is post-smoothing by one or more steps of the iterative method.

The linear system on the coarse grid, if not the coarsest grid, is itself solved by pre-smoothing followed by coarse-grid correction and post-smoothing. We thus have a recursive definition of a $\mathrm{V}$-cycle. The following algorithm, however, describes an iterative implementation requiring only three vectors on each grid. It is easily shown that the storage requirement for all $M+1$ grids is approximately $\frac{4}{3}$ times the requirement for $G_{M}$.

$$
\begin{aligned}
& \text { for } i=M, M-1, \ldots, 1 \quad \text { Downward part of V-cycle } \\
& u_{i}=S\left(b_{i}, u_{i}\right) \quad \text { Pre-smoothing } \\
& r_{i}=b_{i}-A_{i} u_{i} \quad \text { Residual on grid } G_{i} \\
& b_{i-1}=R\left(r_{i}\right) \quad \text { Restriction of } r_{i} \text { to } G_{i-1} \\
& \text { endfor } \\
& u_{i-1}=0 \\
& u_{0}=A_{0}^{-1} b_{0} \\
& \text { for } i=1,2, \ldots, M \\
& r_{i}=I_{n}\left(u_{i-1}\right) \\
& u_{i}=u_{i}+r_{i} \\
& \text { endfor } \\
& u_{i}=S\left(b_{i}, u_{i}\right) \quad \text { Post-smoothing } \quad \text { to } G_{i}
\end{aligned}
$$


Our smoothing operator $S\left(b_{i}, u_{i}\right)$ consists of two steps of the weighted Jacobi method. While theory suggests $w=\frac{2}{3}$ as the optimal weight, we empirically determined that $w=0.88$ was optimal for our test data. The restriction operator $R\left(r_{i}\right)$ computes a grid-point value on the coarse grid as a weighted average of $r_{i}$ values at the grid point and its eight neighbors in the finer grid with weights $\frac{1}{4}$ at the grid point, $\frac{1}{8}$ at the four horizontally and vertically adjacent neighbors, and $\frac{1}{16}$ at the four diagonally adjacent neighbors. (The scheme is modified appropriately for grid points on the boundary.) The interpolation operator $I_{n}$ uses piecewise bilinear interpolation. Unless the domain is a rectangle, the system on the coarsest grid $G_{0}$ is nontrivial, and we use a conjugate gradient method to compute $u_{0}$.

In the full multigrid method the initial solution estimate for the finest grid is obtained by interpolating a solution computed by applying a V-cycle on the coarser grid. In our application the Sobolev gradient computed at each descent step provides a good initial estimate for the subsequent step, with increasing accuracy as the descent iteration nears convergence. We therefore solve each linear system by a sequence of V-cycles on the finest grid. See [Demmel 1997] for further background on the multigrid method.

\section{RESULTS}

We take our domain to be a 10 by 6 rectangle and employ four grid refinements with $k_{x}=5$ and $k_{y}=3$ on the coarsest grid $G_{0}$, so that the finest grid $G$ consists of 80 by 48 cells with mesh widths

$$
h_{x}=h_{y}=\frac{1}{8} .
$$

We begin with material number $\kappa=1$ and constant applied magnetic field $H_{0}=1$. Figures $1-4$ depict the electron densities, induced magnetic fields, and current magnitudes associated with four critical points obtained by varying initial solution estimates. The energy levels $\left(\varphi=\varphi_{G}\right.$ values $)$, specified in the figure captions, constitute a decreasing sequence. Note the similarities between the electron density and magnetic field of each critical point.

At the bottom of the same figures we show also the corresponding currents as vector fields. The presence of counter-currents is clearly demonstrated in this set of figures. For each critical point, the degree of the wave function $u$ on the boundary was computed (by counting the number of cycles of the phase angle). This computation verified that the number of vortices agrees with the number of local extrema apparent in the plots -1 to 4 . The degree0 critical point corresponding to

$$
u=0, \quad \nabla \times A=H_{0}
$$

(not depicted) has energy value 15. The increasing complexity of the electron densities appears similar to that of a sequence of eigenfunctions of a linear operator (Laplacian) associated with an increasing sequence of eigenvalues (energy levels).

We now alter the domain with the removal of a 2 by 2 square from the center. In this case we found five distinct critical points with energy levels 12.65, $11.26,11.17,10.83$, and 10.45. However, we display only the first and last. Figures 5 and 6 depict the electron densities, magnetic fields, magnitudes of current, and currents. Note that the magnetic field is constant in the hole. This property agrees with theory and adds to our confidence that the computed results are accurate.

For our final test domain, we remove two symmetrically placed squares from the 10 by 6 rectangle. We found only three critical points in this case: energy levels 10.44, 9.38, and 8.44. The electron densities, magnetic fields, current magnitudes, and currents for the first and last solutions are depicted in Figures 7 and 8.

For our final tests we increased the material number to $\kappa=2$ on the one-hole domain, first with $H_{0}=1$ (Figure 9) and then with $H_{0}=2$ (Figure 10). We computed just one critical point in this case. The increase in $\kappa$ suppresses the counter-currents which are then restored by the increase in $H_{0}$. This demonstrates the ability to control the presence of counter-currents by varying parameter values. The relationship between parameter values and solutions appears, however, to be quite complex.

\section{ACKNOWLEDGEMENTS}

We express our appreciation to Jacob Rubinstein, who introduced us to the subject of superconductivity and has provided us with substantial advice throughout our investigations. 
528 Experimental Mathematics, Vol. 9 (2000), No. 4
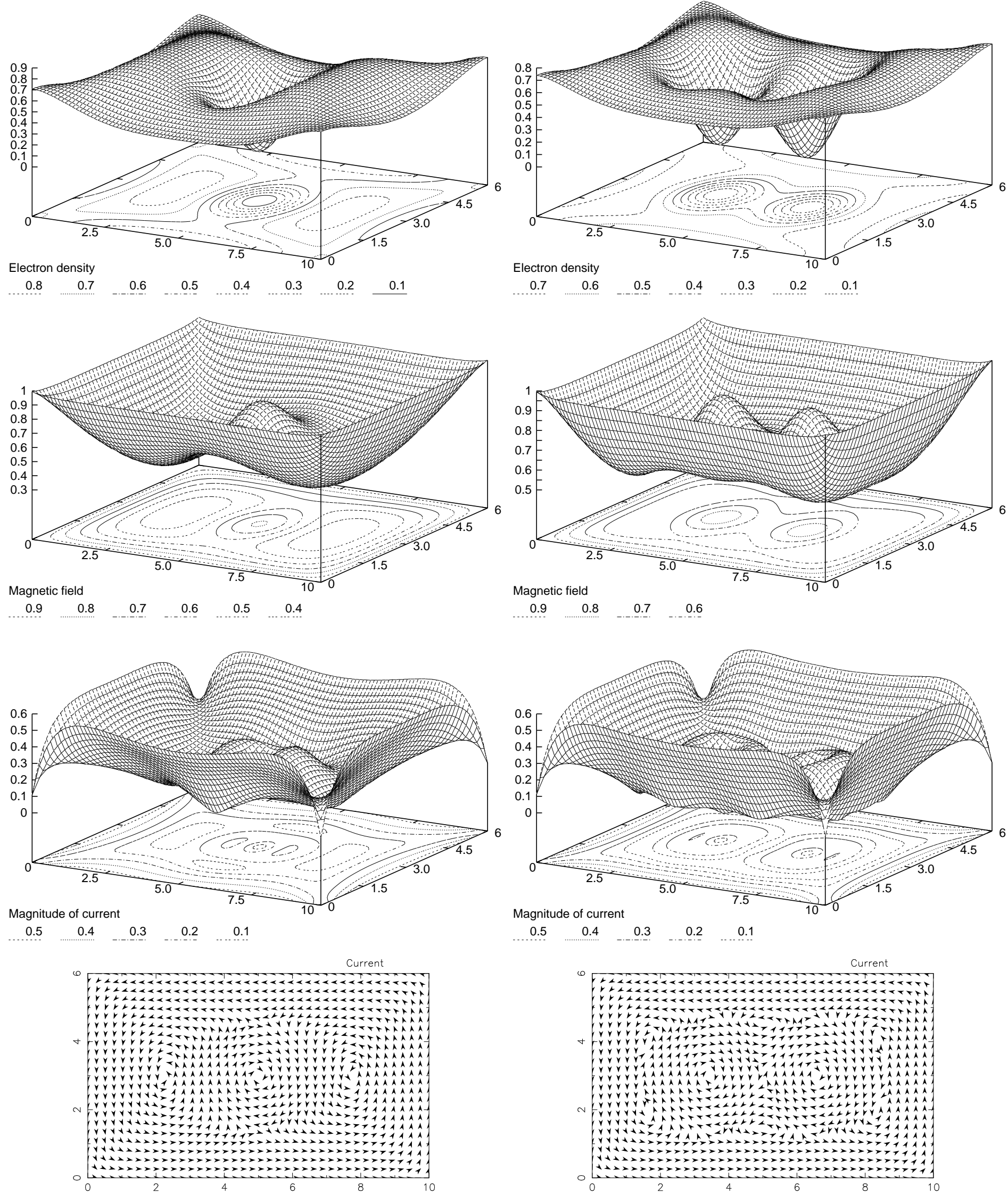

FIGURE 1. Solution 1: $\varphi=13.75$.

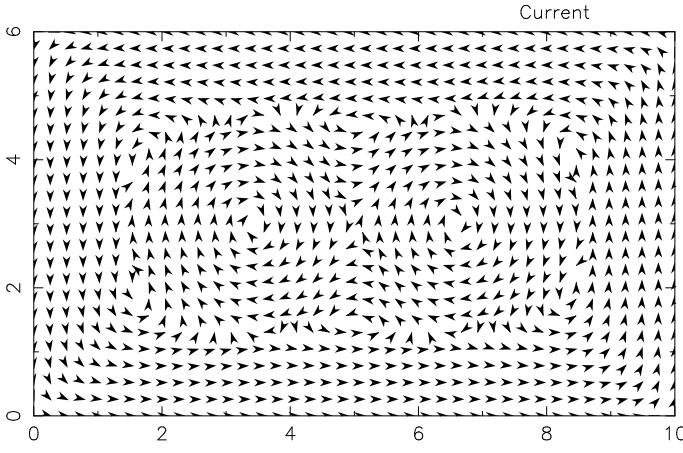

FIGURE 2. Solution 2: $\varphi=12.58$. 

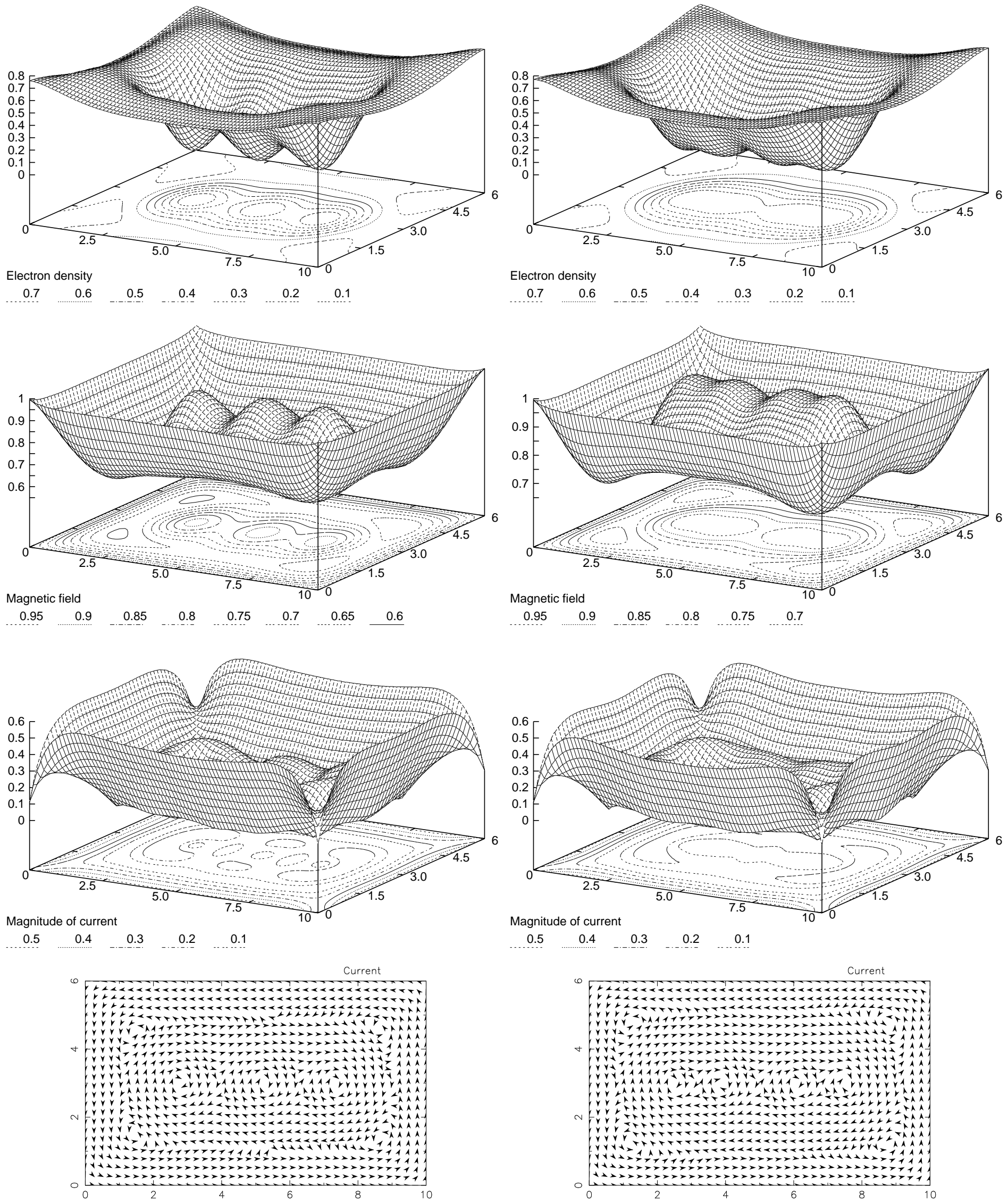

FIGURE 3. Solution 3: $\varphi=11.94$.

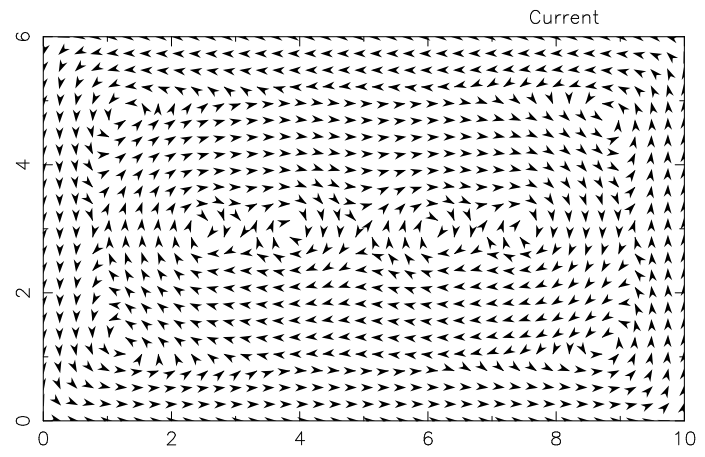

FIGURE 4. Solution 4: $\varphi=11.69$. 
530 Experimental Mathematics, Vol. 9 (2000), No. 4
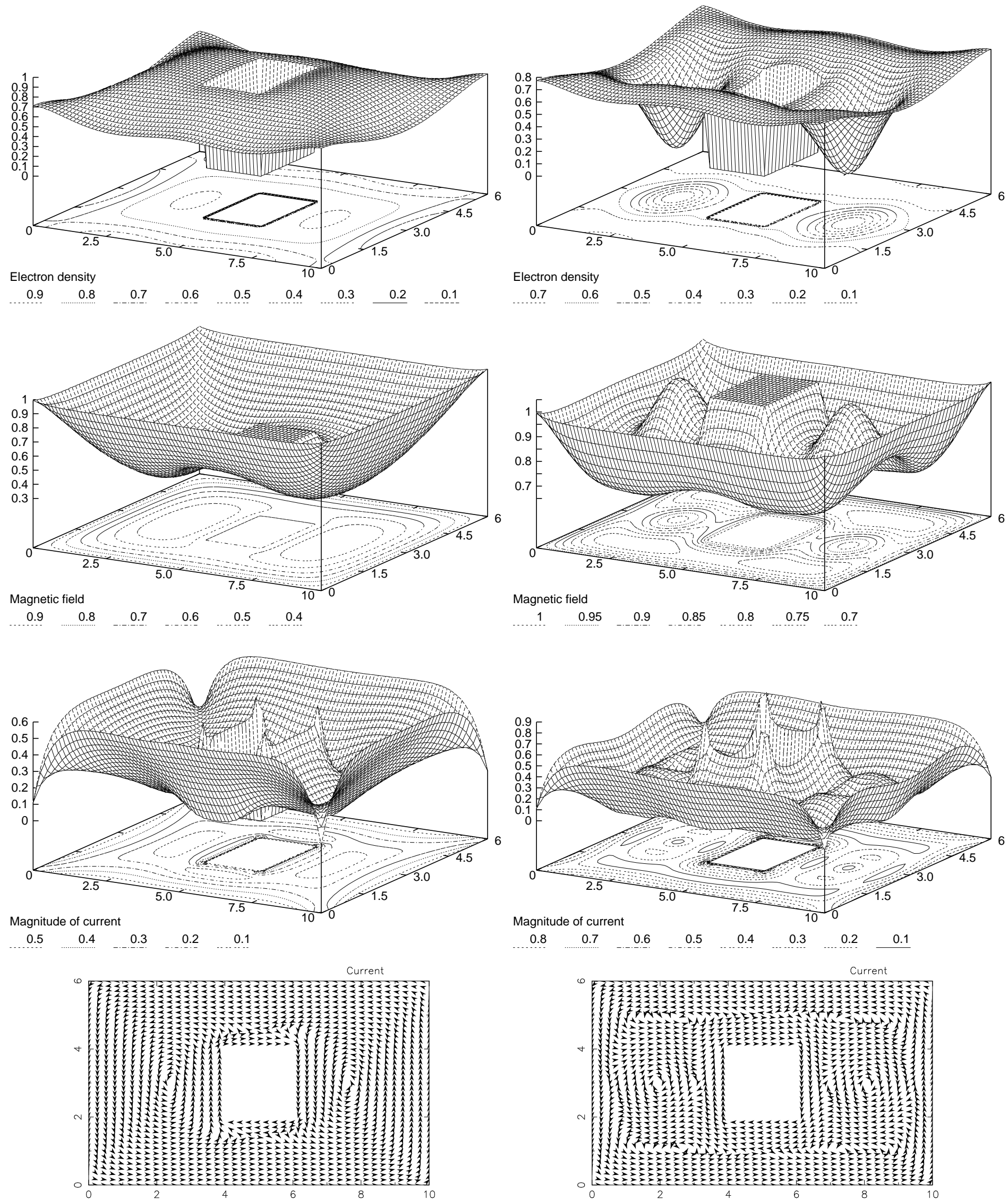

FIGURE 5. One-hole solution 1: $\varphi=12.65$.

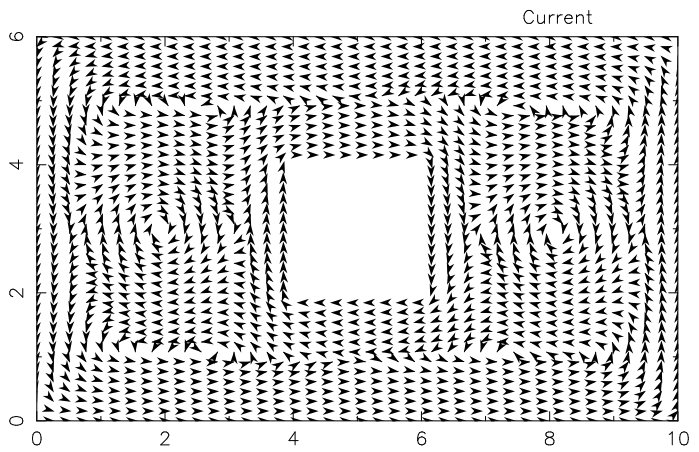

FIGURE 6. One-hole solution $5: \varphi=10.45$. 

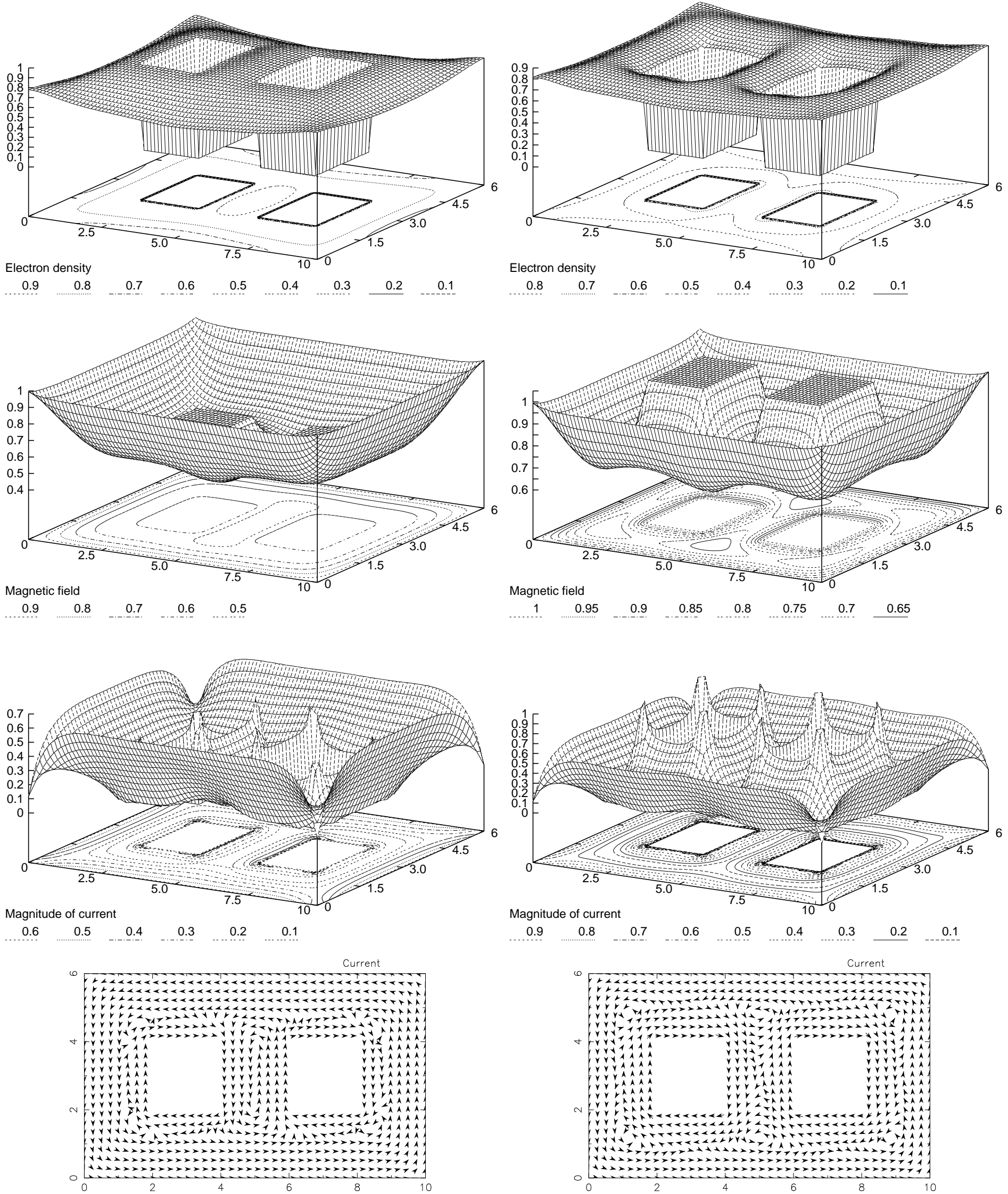

FIGURE 7. Two-hole solution 1: $\varphi=10.44$.

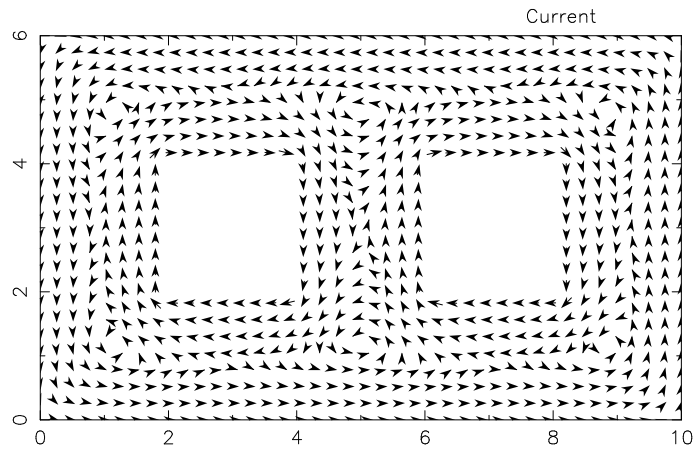

FIGURE 8. Two-hole solution $3: \varphi=8.44$. 
532 Experimental Mathematics, Vol. 9 (2000), No. 4
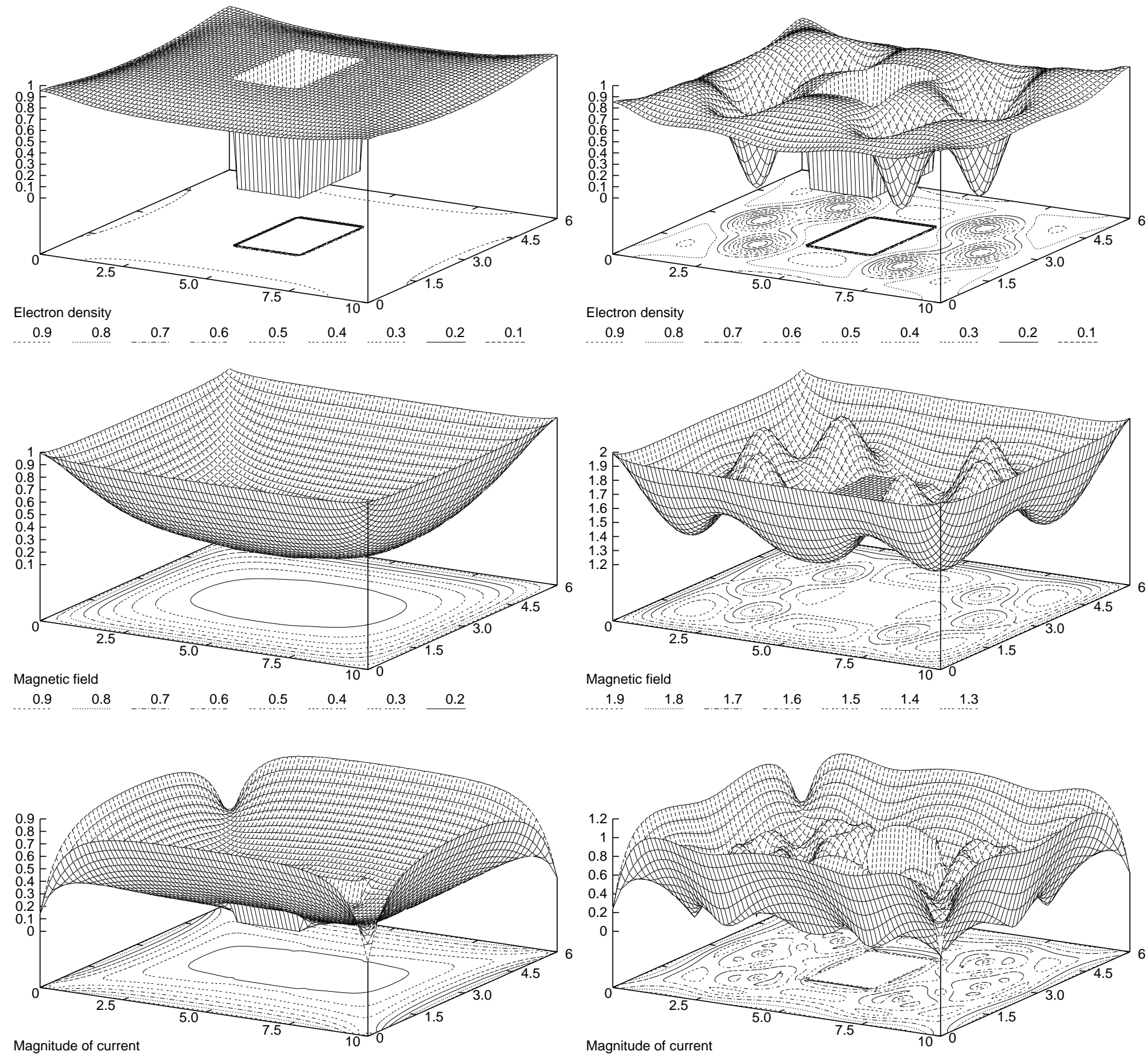

\begin{tabular}{llllllll}
0.8 & 0.7 & 0.6 & 0.5 & 0.4 & 0.3 & 0.2 & 0.1 \\
\hline
\end{tabular}
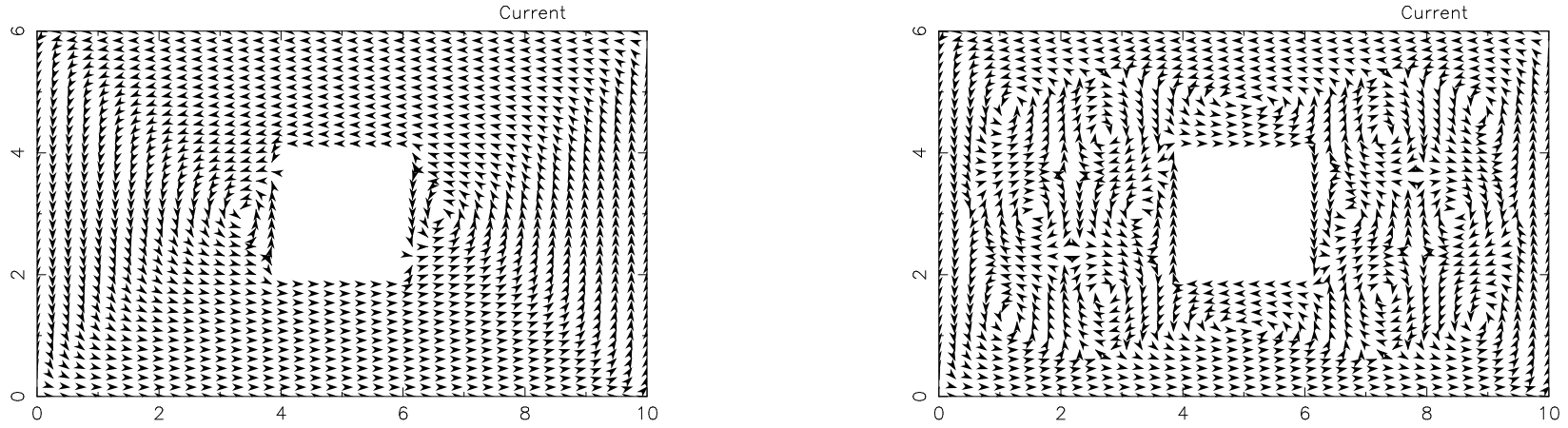

FIGURE 9. One-hole solution 1: $\kappa=2, H_{0}=1, \varphi=20.29$.

FIGURE 10. One-hole solution 1: $\kappa=2, H_{0}=2, \varphi=35.49$. 


\section{REFERENCES}

[Bethuel et al. 1994] F. Bethuel, H. Brézis, and F. Hélein, Ginzburg-Landau vortices, Progress in Nonlinear Diff. Eq. Appl. 13, Birkhäuser, Boston, 1994.

[Brent 1973] R. P. Brent, Algorithms for minimization without derivatives, Prentice-Hall, Englewood Cliffs, NJ, 1973. Errata in Math. Comput., 29, (1975), p. 1166 .

[Comte and Mironescu 1995] M. Comte and P. Mironescu, "Étude d'un minimiseur de l'énergie de Ginzburg-Landau près de ses zéros", C. R. Acad. Sci. Paris Sér. I Math. 320:3 (1995), 289-293.

[Demmel 1997] J. W. Demmel, Applied numerical linear algebra, SIAM, Philadelphia, PA, 1997.

[Du et al. 1992] Q. Du, M. D. Gunzburger, and J. S. Peterson, "Analysis and approximation of the Ginzburg-Landau model of superconductivity", SIAM Rev. 34:1 (1992), 54-81.

[Du et al. 1995] Q. Du, M. D. Gunzburger, and J. S. Peterson, "Computational simulation of typeII superconductivity including pinning phenomena", Physical Review B 51:22 (1995), 16194-16203.

[Fleckinger-Pellé et al. 1998] J. Fleckinger-Pellé, H. G. Kaper, and P. Takáć, "Dynamics of the GinzburgLandau equations of superconductivity", Nonlinear Anal. 32:5 (1998), 647-665.
[Jaffe and Taubes 1980] A. Jaffe and C. Taubes, Vortices and monopoles: structure of static gauge theories, Prog. in Physics 2, Birkhäuser, Boston, 1980.

[Mu and Huang 1998] M. Mu and Y. Huang, "An alternating Crank-Nicolson method for decoupling the Ginzburg-Landau equations", SIAM J. Numer. Anal. 35:5 (1998), 1740-1761.

[Neuberger 1997] J. W. Neuberger, Sobolev gradients and differential equations, Lecture Notes in Math. 1670, Springer, Berlin, 1997.

[Neuberger and Renka 1997] J. W. Neuberger and R. J. Renka, "Numerical calculation of singularities for Ginzburg-Landau functionals", Electron. J. Differential Equations 1997:10 (1997), 1-4.

[Neuberger and Renka 1999] J. W. Neuberger and R. J. Renka, "Sobolev gradients and the Ginzburg-Landau functional", SIAM J. Sci. Comput. 20:2 (1999), 582590 .

[Rubinstein 1998] J. Rubinstein, "Six lectures on superconductivity", pp. 163-184 in Boundaries, interfaces, and transitions (Banff, AB, 1995), edited by M. C. Delfour, Amer. Math. Soc., Providence, RI, 1998.

[Serfaty 1999] S. Serfaty, "Stable configurations in superconductivity: uniqueness, multiplicity, and vortexnucleation", Arch. Ration. Mech. Anal. 149:4 (1999), 329-365.

[Shafrir 1995] I. Shafrir, " $L^{\infty}$-approximation for minimizers of the Ginzburg-Landau functional", $C . R$. Acad. Sci. Paris Sér. I Math. 321:6 (1995), 705-710.

J. W. Neuberger, Department of Mathematics, University of North Texas, Denton, TX 76203-5116 (jwn@unt.edu)

R. J. Renka, Department of Computer Science, University of North Texas, Denton, TX 76203-1366

(renka@cs.unt.edu)

Received May 5, 1999; accepted in revised form March 31, 2000 